\title{
Complex Droplets and Interacting Bubbles Receive Video Prize
}

\section{An annual APS video prize went to research on the breakup of fluid} filaments, the jet produced by a bubble pair, and the surprising patterns that a 2D flow can create.

\section{By David Ehrenstein}

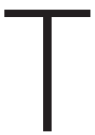

he APS Division of Fluid Dynamics has announced the 2021 winners of its annual Gallery of Fluid Motion video and poster contest. Below are the video winners of the Milton van Dyke Award, which recognizes the three top videos and the three top posters.

\section{Oil and Water Team Up}

"Droplets are everywhere," says Alban Sauret of the University of California, Santa Barbara (UCSB), from rain to spray paint, to
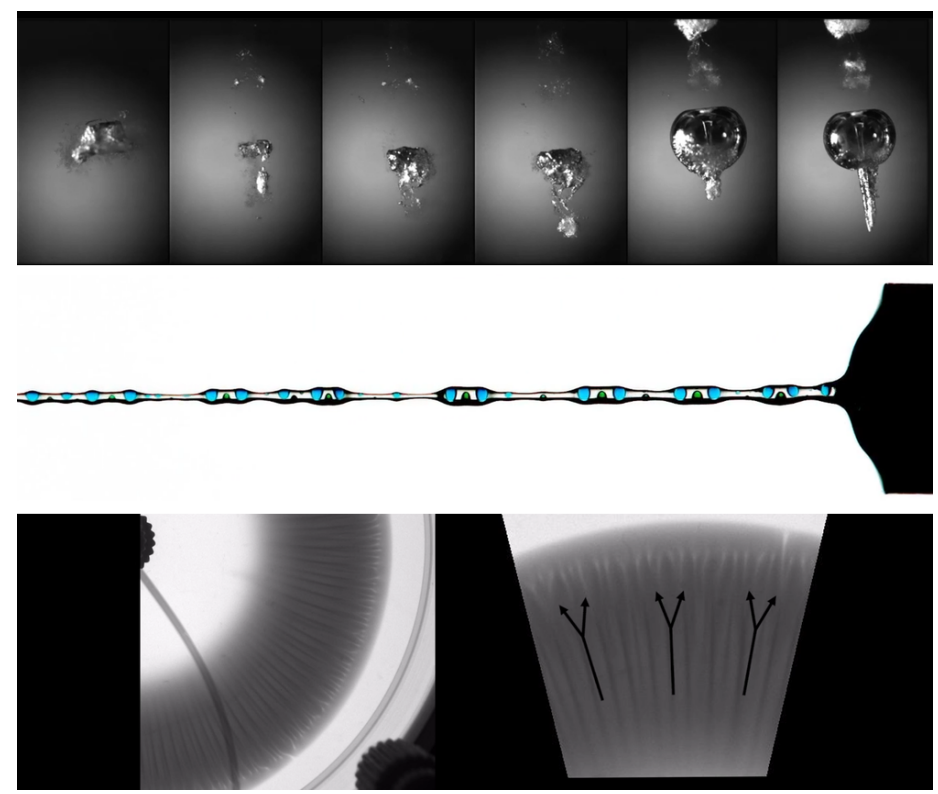

Credit: A. Mishra/V. Thievenaz/Y. Stergiou

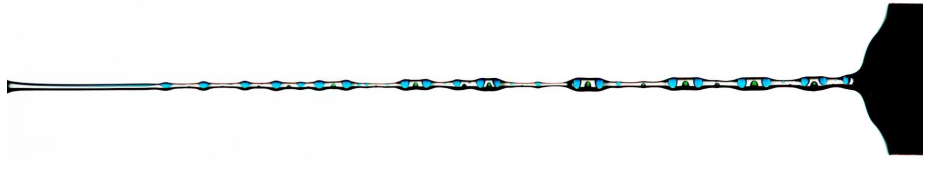

A liquid filament containing two different liquids breaks up into droplets in a way that is affected by both components. The video of these breakups is slowed by about 100 times. (More information.) Credit: V. Thievenaz/UCSB

sneezes. Often these droplets emerge from the breakup-or "atomization"-of a large volume of fluid. Understanding this behavior could, for example, help researchers generate the number and size of droplets to efficiently coat a surface with paint or a field with fertilizer. To observe atomization up close, Sauret and UCSB colleague Virgile Thievenaz have developed a technique in which they place a small amount of fluid in the gap between two rods and then rapidly pull the rods apart. A filament of fluid appears and then breaks up into droplets.

Their latest twist on this experiment is to include two different 
fluids-a small droplet of water inside a larger drop of viscous oil. To the duo's surprise, the water accelerated the breakup of the oily filament and affected the sizes of the final droplets, even with only $1 \%$ water in the fluid. Meanwhile, the oil influenced the water by causing it to stretch out much longer than usual before breaking into droplets. "We weren't expecting that there would be such a drastic change with just a very small amount [of water]," Sauret says. He says that pesticide companies, for example, might be able to reduce unwanted drift of their product by embedding a small amount of another fluid that reduces the number droplets with the smallest diameters, the ones most likely to be picked up by wind.

\section{Interacting Bubbles Create a Jet}

Cavitation bubbles often form when a solid object moves rapidly through fluid, as in a rotating ship propeller.

Researchers have often studied these clouds of bubbles or looked at individual bubbles, but much less research has focused on the interactions between bubbles, says graduate student Arpit Mishra of the Indian Institute of Technology Kharagpur, India. He worked with Claire Bourquard of the Swiss Federal Institute of Technology (ETH Zurich) to develop a laser-based system for creating two millimeter-sized bubbles in water. The researchers wanted to produce the bubbles at two specific times and positions in order to control their interactions. "The timing was very challenging," says Bourquard, and "we didn't know if it was going to work."

But it did work, and Mishra, Bourquard, and their colleagues observed something unexpected: With the right time and distance between the bubbles, a powerful jet of water vapor shot out of the second bubble as it collapsed. Based on experiments and simulations, the team learned that the jet is produced by a "slingshot" effect. The first bubble collapses and draws part of the second bubble toward it, like an elastic band being stretched. When the second bubble collapses, the outstretched portion comes rebounding back with enough energy to push vapor out the other side of the bubble in a jet.

These bubble-generated microjets could have potential medical applications. Indeed, cavitation bubbles are currently used for dental cleaning and surgical cutting. The team explored the possibility of using microjets to puncture biological tissue without the need for a needle. Their video demonstrates that a bubble-generated microjet can poke through a

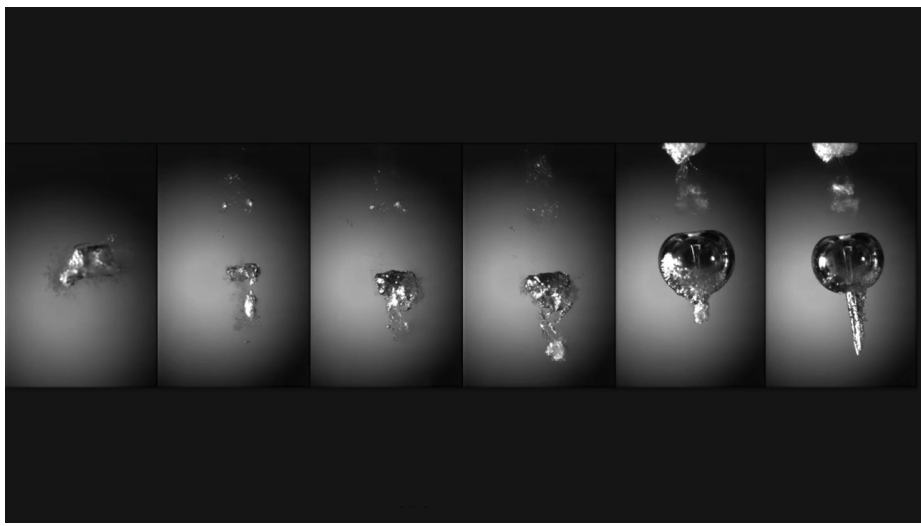

Researchers produced two bubbles near each other at slightly different times and showed that the interaction between the two produces a jet of vapor when the second bubble collapses. The videos are shown at 15 frames per second (fps), so as indicated by the recording rates given in $\mathrm{fps}$, they are slowed down by about 7000 to 700,000 times. (More information.)

Credit: A. Mishra/Indian Institute of Technology Kharagpur

5-millimeter-thick layer of agarose, a gel they use as a stand-in for living tissue.

\section{D Fluid Flow Isn't So Simple}

One way to dispose of carbon dioxide $\left(\mathrm{CO}_{2}\right)$ and help fight climate change is to inject $\mathrm{CO}_{2}$-rich liquid into calcium-rich soil and create stable calcium carbonate. Anne De Wit of the Free University of Brussels (ULB) and her colleagues modeled this carbon sequestration process theoretically and experimentally a few years ago. Their model assumed a simple 2D geometry in which the $\mathrm{CO}_{2}$-rich liquid is injected into the center of a horizontal disk containing a calcium-rich solution. But "even such a simple situation is very complex," says De Wit.

The complexity that the researchers uncovered involved convection rolls, vertical loops in which heavier fluid sinks and lighter fluid floats. The presence of these flow patterns implied that the $2 \mathrm{D}$ assumption was not completely accurate, as buoyancy and gravity forces were acting in the vertical direction. To explore the role of this 3rd dimension, De Wit's colleagues Karin Schwarzenberger and Yorgos Stergiou of the Helmholtz Center in Dresden, Germany, ran experiments aboard an airplane that alternated between microgravity (near 


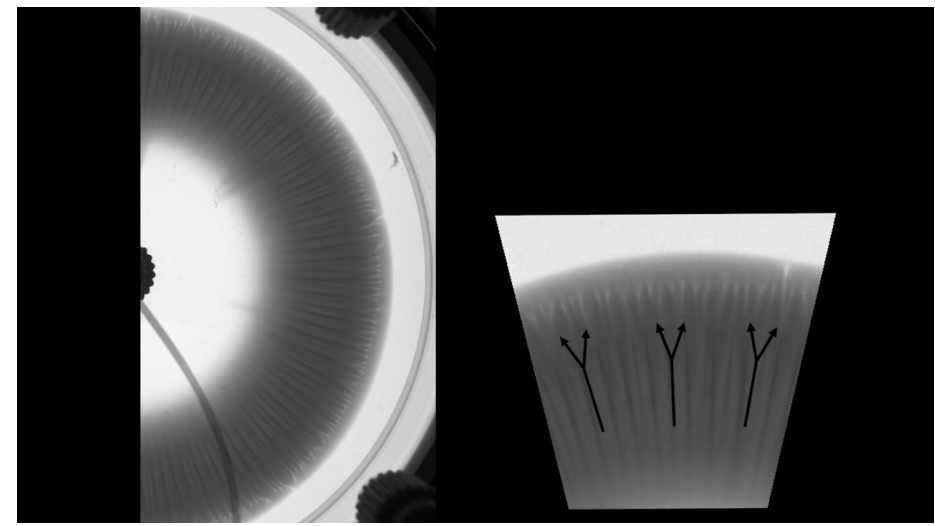

A seemingly $2 \mathrm{D}$ flow exhibits complex patterns that imply $3 \mathrm{D}$ convective flows. Microgravity experiments confirm that convection is at work, as the patterns disappear without gravity to drive convection. Some of these videos are sped up by as much as 4 times. (More information.)

Credit: Y. Stergiou/Helmholtz-Zentrum Dresden-Rossendorf zero g) and hypergravity (2g). As shown in their video, the convection rolls disappeared at zero g, proving that these flow features resulted from gravity and buoyancy. The hypergravity phase produced even more complex patterns that the team is still analyzing.

De Wit points out that understanding this type of system, a so-called reaction-diffusion problem with injection, has applications in a wide range of fields beyond carbon sequestration. For example, a COVID-infected person can be thought of as the source of an outward-spreading wave of infection.

David Ehrenstein is a Senior Editor for Physics. 